\title{
Does a Nurse Led Surveillance Program Using a Verbal Reminder Intervention Impact Catheter-Associated Urinary Tract Infection Rates?
}

\author{
Rachel Akpom* \\ Department of Nursing, Mary Starke Harper Geriatric Psychiatry Center, Alabama, USA
}

*Corresponding author: Rachel Akpom, Department of Nursing, Mary Starke Harper Geriatric Psychiatry Center, Alabama, USA.

Received Date: April 08, 2020

Published Date: July 06, 2020

\begin{abstract}
Catheter-Associated Urinary Tract Infection (CAUTI) is a common occurring hospital-acquired infections (HAIs) secondary to the use of indwelling urinary catheters (IUCs). This DNP project evaluated the impact of a nurse-led surveillance program using a verbal reminder intervention on CAUTI rate, IUC days, and nurses' knowledge of the Centre for Disease Control (CDC) on CAUTI guidelines in a medical surgical unit in a large hospital in Texas. This study used a pre and post-test design. Control group data were obtained from a three-month retrospective CAUTI rate and IUC duration information from electronic medical records of discharged patients. The period reviewed was from October 2017 to December 2017. During the intervention period, nurses implemented a nurse-led surveillance program using a verbal reminder intervention to prompt timely removal of indwelling urinary catheters. Data collection included CAUTI incidents, IUC days, and improvement on nurses' knowledge of the CDC CAUTI guidelines before and after implementation. Descriptive statistics of CAUTI incidents and IUC days were computed and analysed. The results show a decrease in CAUTI incidents from 1 for the pre-intervention group to 0 for the post-intervention group. The number of patients with IUCs for three days decreased from 36(30.51\%) before implementation to 9 (7.63\%) after implementation. T-test analysis was performed for Nurses' knowledge using Minitab 18 Statistical Data Analysis Software. Nurses' knowledge test revealed a significant improvement in mean scores from $77 \%$ before implementation to $91.5 \%$ after implementation.
\end{abstract}

Keywords: Catheter associated urinary tract infection (CAUTI); Lowa model; Medical-surgical unit; Indwelling urinary catheter; Nurse-led surveillance

\section{Introduction}

Catheter-Associated Urinary Tract Infection (CAUTI) is a frequently occurring urinary tract infection among hospitalized patients. CAUTI is preventable, yet it continues to be a frequently occurring infection incident among hospitalized patients worldwide, because of prolonged duration of IUC being constantly in physical contact with the bladder. The constant physical touch of IUC with bladder causes increase in bacteria accumulation and transmission to the bladder; resulting in CAUTI [1-5]. As a result, patients experience prolonged hospitalization, and increased healthcare costs. CAUTI increases the risk of patient's discomfort, health complications, and can lead to sepsis and death. It is vital for the nurse to understand the importance of evaluating the indwelling catheter days and understanding the importance of having these indwelling urinary devices removed if no longer necessary for medical care.

Nurses spend most of their working hours with patients at the bedside and nurses were the best modality to implement this intervention to make a positive impact in this organization. The use of a nurse-led surveillance program using a verbal reminder intervention system to monitor indwelling urinary catheters 
was a daily intervention that monitored the duration of IUCs and reminded physicians of the presence of indwelling catheters (ICs). This interventional quality improvement DNP project ensured timely discontinuation of ICs when the use of ICs was no longer required for the patient's medical condition and treatment. Nurses' active participation in preventing the risk of CAUTI had an impact on patients' outcomes which reduced patients' discomfort, prolonged length of hospitalization, unnecessary infection, and even death. Hospitals have benefitted from this intervention since the facility did not experience loss of financial compensation for patient care related to CAUTI. The focus of this project was to evaluate the effect of a nurse-led surveillance program using a verbal reminder intervention on catheter duration and CAUTI rate.

\section{PICOT Question}

In hospitalized adult patients $>18$ years of age, does the use of a nurse-led surveillance program using a verbal reminder intervention system of the indwelling urinary catheter compared to organization standard protocol affect CAUTI rates over a 3-month period?

\section{Research Questions}

The research questions for this study were:

- Did CAUTI rates decrease after implementation of a nursing staff education program using an evidence-based nurse-led surveillance verbal reminder system?

- $\quad$ Did IUC days decrease after implementation of a nursing staff education program using an evidence-based nurse-led surveillance verbal reminder system?

- Did implementation of a nursing staff education on CDC guidelines helped reduce CAUTI rates as evidenced by improved scores on the nursing staff post-test?

\section{Purpose}

The purpose of this DNP project was to implement and study the effect of a nurse-led surveillance program using a verbal reminder intervention of IUC on CAUTI rate in a medical-surgical unit. The three outcome objectives of this quality improvement project were the following:

- to reduce urinary catheter infections,

- to reduce urinary catheter days, and

- to evaluate nursing knowledge using CDC CAUTI guidelines.

To accomplish these project objectives, nurses played a significant role in the process of this project.

\section{Conceptual and Theoretical Framework}

The conceptual framework used to guide this project was the Iowa Model of Evidence-based Practice (EBP) [3] for healthcare excellence. The Iowa Model is one of the most frequently used models for evidence-based research studies by healthcare providers to produce positive patient outcomes. Nurses are the frontline healthcare providers. They spend most of their professional hours in providing direct care to patients, which includes IUC management. As a result, it is crucial that nurses are well educated on EBP related to IUC management to ensure healthcare excellence, and to improve patient outcomes. Additionally, it was important to encourage Nurses' active participation in IUC management through surveillance and verbal reminder system to prevent CAUTI incidents.

\section{Relevance to Nursing Practice}

The Iowa Model was relevant to nursing practice because it emphasized the use of Evidence-Based Practice (EBP) by healthcare providers. Nurses were at the frontline of patient care. Therefore, Nurses' consistent implementation of EBP in IUC management improved patient outcomes. The goal of this project was to decrease CAUTI rate to 0 or below the CDC national benchmark of 1.07/1000 IUC days on the proposed unit. A nurse-led surveillance program using a verbal reminder intervention of the presence of IUC to physicians is an intervention based on EBP, and it was consistent with the Iowa Model for EBP to promote excellence in healthcare.

\section{Setting}

The setting of this DNP project was in a medical-surgical unit in one of the hospitals in Texas. The unit provided care to all medical-surgical patients regardless of the nature of their surgical procedure. The nurses working in the medical surgical unit were Associate and BSN prepared Registered Nurses. The unit has a daily average census of 4 to 8 patients with indwelling urinary catheters. The daily average population of patients was 20 to 28 with eight licensed nursing staff. The patients' ages are 30 years and above.

\section{Patient Selection}

A computer-generated random sample method was used to select 118 patients' charts for retrospective chart review of patients who had indwelling urinary catheters during hospitalization between October and December 2017. The urine culture results, IUC insertion date, and discontinuation date were reviewed during these same dates. The durations of IUCs were collected using the IUC insertion dates and discontinuation dates identified in the chart. These datasets were used to compare to the CAUTI data collected during the surveillance intervention period of this study. A 3-month retrospective CAUTI data was collected from the electronic medical records of 118 patients with the assistance of the infection control nurse using the retrospective CAUTI data collection form for patients admitted between October 1st, 2017 and December 31st, 2017. G-Power analysis indicates that a minimum of 105 sample size would be required respectively to achieve a .95 level of power.

\section{Intervention}

At the initiation of this project, a 1-week implementation trial was performed to ensure that the registered nurses understood 
the forms and process of this study. The purpose of having the implementation trial was to ensure validity and reliability of the process. During the 1-week period, the unit registered nurses had one-on-one consultations with the researcher for clarifications. The researcher observed the unit nurses' use of the nurse-led surveillance program using a verbal intervention; the researcher provided feedback at the time as needed. During the intervention period, the registered nurses were responsible for implementation of the nurse-led surveillance program using a verbal reminder intervention daily on the unit. Additionally, the registered nurses were responsible for collecting CAUTI data daily and were also responsible for ensuring removal of the ICs after the physician's order was obtained to discontinue the IUCs and they were responsible during the process to document the intervention that occurred on a daily IUC surveillance forms. At the completion of this study, to ensure sustainability, the nurse manager was encouraged to ensure that quarterly in-service training will be conducted in the future to ensure that the knowledge, process, and procedures for the nurse-led surveillance program using a verbal reminder intervention will be sustained.

\section{CAUTI Surveillance Program}

A nurse-led surveillance program using a verbal reminder intervention was implemented in a medical surgical unit involving Registered Nurses' daily routine on hospitalized patients whose care required the use of IUCs. Registered Nurses reminded physicians of the presence of IUCs and requested for revaluation of the continuous use of the IUCs. The nurse-led surveillance program using a verbal reminder intervention prompted physician's order for discontinuation of ICs.

\section{Results}

The result showed that out of the 118 patients in preintervention group, three patients $(2.54 \%)$ had IUCs for one day, 79 patients $(66.95 \%)$ had IUCs for two days while 36 patients $(30.51 \%)$ had IUCs for three days. For post-intervention group, the number of patients with IUC duration of 1 day was 4 (3.39\%). The number of patients with IUC duration of 2 days was 105 (88.98\%) and the number with IUC duration of 3 days was 9 (7.63\%). A descriptive statistic showed a decrease in the number of patients who had IUC duration at 3 days from 36 to 9 respectively however, the rates were higher in 1 and 2 IUC days in the post-intervention groups. Compared to total sample, the number of patients with IUC duration of 3 days decreased from $30.51 \%$ in pre-intervention group to $7.63 \%$ in post-intervention group.

\section{Summary}

The use of a nurse-led surveillance program using a verbal reminder intervention was implemented during the post intervention period. IUC discontinuations increased in the post intervention group. CAUTI incidents decreased in post intervention sample. CAUTI rate for all patients' electronic medical records reviewed before implementation of this project was $3.71 \%$. When compared to post-implementation CAUTI incident, the unit's CAUTI rate decreased from $3.71 \%$ to $0 \%$. To evaluate knowledge level of Registered Nurses on CDC Guidelines on CAUTI the nurses were given a pre- and post-tests about CAUTI. The pre-test scores were compared to post-test scores for each nurse, during this project. Minitab 18 statistical data analysis software program was used to analyze the scores. The results showed a statistically significant difference $(\mathrm{p}<=0.05)$ in the scores of nurses between pre-test and post-test scores.

\section{Acknowledgement}

I am grateful to my mentor and members of my dissertation committee who participated in this project.

\section{Conflicts of Interest}

Author does not have any conflict of interest.

\section{References}

1. Durant DJ (2017) Nurse-driven protocols and the prevention of catheter-associated urinary tract infections: A systematic review. Am J Infect Control 45(12): 1331-1341.

2. Institute for Healthcare Improvement (2011) How-to-guide: Prevent catheter-associated urinary.

3. Titler MG (2008) The evidence for evidence-based practice implementation.

4. Wang PT, Lin HY, Lin YT, Shin WH, Chien LY, et al. (2017) Using an indicator-based reminder of catheter removal to effectively decrease catheter-associated urinary tract infections in general medical patients. The Journal of Nursing 64(1): 70-79.

5. Yatim J, Wong KS, Ling ML, Tan SB, Tan KY, et al. (2016) A nurse-driven process for timely removal of urinary catheters. International Journal of Urological Nursing 10(12): 167-172. 